\title{
The hemostatic mechanism of "Treated the Spleen" therapy on immune thrombocytopenia based on the characteristics of vasoactive factors
}

\author{
Yayue Zhang ${ }^{1 \#}$, Min Jiang ${ }^{2 \#}$, Jia Wang ${ }^{3}$, Jieyin Gao ${ }^{4}$, Ming Guo ${ }^{4}$, Junxia Liu ${ }^{4}$, Xinyi Chen ${ }^{1}$, Haiyan Lang ${ }^{4}$ \\ ${ }^{1}$ Department of Oncology and Hematology, Dongzhimen Hospital Affiliated to Beijing University of Chinese Medicine, Beijing, China; ${ }^{2}$ Department \\ of Oncology, Dongfang Hospital Affiliated to Beijing University of Chinese Medicine, Beijing, China; ${ }^{3}$ Henan Cancer Hospital, Zhengzhou, China; \\ ${ }^{4}$ Department of Hematology, Dongfang Hospital Affiliated to Beijing University of Chinese Medicine, Beijing, China \\ Contributions: (I) Conception and design: Y Zhang, M Jiang, H Lang; (II) Administrative support: X Chen, H Lang; (III) Provision of study materials \\ or patients: J Wang and J Gao; (IV) Collection and assembly of data: M Guo, J Liu; (V) Data analysis and interpretation: Y Zhang, M Jiang; \\ (VI) Manuscript writing: All authors; (VII) Final approval of manuscript: All authors. \\ "These authors contributed equally to this work. \\ Correspondence to: Haiyan Lang. Department of Hematology, Dongfang Hospital Affiliated to Beijing University of Chinese Medicine, 6 \\ Fangxingyuan 1st Block, Fengtai District, Beijing 100078, China. Email: julietlang@126.com.
}

Backgroundk To explore the effect mechanism of "treat the spleen" therapy on immune thrombocytopenia (ITP) based on the characteristics of vasoactive factors.

Methods: The ITP mice model was established by passive immunomodeling. 120 successfully modeled $\mathrm{BALB} / \mathrm{c}$ mice were randomly divided into 6 groups: normal group, model group, prednisone group, Guipi Decoction group, Jianpi Yiqi group, and Jianpi Shexue group. These mice were treated with medicine for 16 days. After treatment, the platelet (PLT) counts and the degree of bleeding were evaluated, and the serum ET-1, NO, NOS3, TXA2, PGI2, vWF, VCAM-1, and TM contents of the model mice were detected by enzyme-linked immunosorbent assay (ELISA).

Results: The PLT counts in peripheral blood of mice in each experimental group significantly decreased compared with that in the normal group $(\mathrm{P}<0.01)$. On the 8 th day after commencing administration, compared with the model group, PLT counts of mice in each experimental group significantly increased $(\mathrm{P}<0.01)$. Before administration, all groups had different bleeding tendencies except for the normal group. On the 6th and 8th day of drug intervention, compared with the model group, the bleeding grade of treated groups was significantly decreased $(\mathrm{P}<0.01)$. The values of ET-1, vWF, and VCAM-1 in each experimental group significantly decreased compared with that in the normal group, while the TXA2 values were up-regulated compared with that in the normal and model groups $(\mathrm{P}<0.01)$. The values of NO and TM in each experimental group significantly decreased compared with that in the normal group $(\mathrm{P}<0.05)$. In addition to the Guipi Decoction group, the NOS3 values in each group were significantly decreased compared with that in the normal group $(\mathrm{P}<0.05)$. The PGI2 values of the "treat the spleen" groups were significantly decreased compared to the normal group $(\mathrm{P}<0.01)$.

Conclusions: In addition to increasing the PLT counts in peripheral blood of ITP model mice to achieve a hemostatic effect, the "treat the spleen" recipes up-regulated the levels of TXA2 and VCAM-1, while down-regulating the levels of PGI2 and TM. Therefore, balancing the procoagulant and anticoagulant factors might be one of the effective mechanisms of hemostasis.

Keywords: Treating the spleen; immune thrombocytopenia (ITP); vascular active factor

Submitted Feb 04, 2021. Accepted for publication Apr 21, 2021.

doi: 10.21037/apm-21-765

View this article at: http://dx.doi.org/10.21037/apm-21-765 


\section{Introduction}

Immune thrombocytopenia (ITP) is a common hemorrhagic blood disorder. The main clinical manifestations of ITP are bleeding of the skin, mucous membrane, gingiva, and nasal mucosa, hematuria, hematochezia or menorrhagia, or prolonged menstrual period. In severe cases, there may be bleeding from the vital organs. The pathogenesis of ITP is not completely clear, and the currently recognized pathogenesis is a multi-factor autoimmune dysfunction $(1,2)$. According to the theory of traditional Chinese medicine (TCM), combined with the clinical characteristics, its onset is due to spleen qi deficiency leading to an inability to control the blood (3). Therefore, "treating the spleen" is the basic principle of treating ITP with TCM $(4,5)$.

An animal model of ITP was replicated by passive immune modeling on the basis of previous clinical and experimental studies (6-8), which is also known as anti-mouse platelet serum modeling method. The preparation of guinea pig antiplatelet serum (GP-APS) resulted in increased platelet destruction and decreased platelet count in mice. According to the TCM criteria of spleen qi deficiency and splenic failure in controlling blood, the ITP model mice established by the passive immune modeling method of injecting APS have the syndrome characteristics of "splenic failure in controlling blood" similar to the onset of human ITP. The peripheral blood platelet (PLT) count was used for bleeding classification of the model mice and serum levels of endothelin-1 (ET-1), thromboxane A2 (TXA2), nitric oxide (NO), nitric oxide synthase 3 (NOS3), prostacyclin (PGI2), thrombomodulin (TM), von Willebrand Factor (vWF), vascular endothelial cells, and vascular cell adhesion molecule-1 (VCAM-1) were used as the detection index. This paper attempted to explore the therapeutic mechanism of "treating the spleen" therapy for the hemostatic effect of ITP from the perspective of the characteristics of vasoactive factors.

We present the following article in accordance with the ARRIVE reporting checklist (available at http://dx.doi. org/10.21037/apm-21-765).

\section{Methods}

\section{Animals}

Guinea pigs weighing $250 \mathrm{~g}$, including 50 males and 50 females, were obtained from Xingping Tianrui experimental animal breeding plant (Xianyang, Shaanxi, China, license No. SCXK(Shaan) 2012-001). Bagg Albino (BALB/c) mice with body weights of 18-22 g, including 140 males and
140 females were obtained from Laboratory Animal Center of Air Force Medical University (Xi'an, Shaanxi, China, license No. SCXK(Shaan) 2014-002). A total of 160 were used for anti-mouse platelet serum (APS) preparation and 120 were used for modeling and mechanism study. The experimental animals were kept in the SPF animal room, the light and dark time was $12 \mathrm{~h} / 12 \mathrm{~h}$. Experiments were performed under a project license (No.: XYLS2019069) granted by ethics board of Medical Ethics Committee of Xi'an Medical College, in compliance with Chinese guidelines for the care and use of animals.

\section{Drugs and reagents}

Chinese medical extracts were obtained from the Xi'an Xinghua Pharmaceutical Research Institute (Shaanxi, China) and were prepared and provided in accordance with the pharmaceutical standards for new Chinese medicine, including Jianpi Shexue extract, consisting of Astragalus membranaceus (Huangqi), Codonopsis pilosula (Dangshen), Poria cocos (Fuling), Rhizoma Atractylodis Macrocephalae (Baizhu), Colla corii asini (Ejiao), Radix rubiae (Qiancao), Radix Glycyrrhizae Preparata (Zhigancao); Jianpi Yiqi extract, consisting of Astragalus membranaceus (Huangqi), Codonopsis pilosula (Dangshen), Poria cocos (Fuling), Rhizoma Atractylodis Macrocephalae (Baizhu), Radix Glycyrrhizae Preparata (Zhigancao); and Guipi Decoction extract, consisting of Ginseng, Rhizoma Atractylodis Macrocephalae (Baizhu), Angelica sinensis (Danggui), Poria cocos (Fuling), Astragalus membranaceus (Huangqi), Arillus Longan (Longyanrou), Polygala tenuifolia (Yuanzhi), Semen Zizyphi Spinosae (Suanzaoren), Elecampane (Muxiang), Radix Glycyrrhizae Preparata (Zhigancao). Prednisone and p-nitrophenylphosphate disodium were purchased from Shanghai Jingchun Biochemical Technology Co., Ltd. Complete and incomplete Freund's adjuvant were purchased from Sigma-Aldrich (St. Louis, MO, USA). Lyophilized enzyme-linked protein A was purchased from Wuhan Boster Biological Technology., Ltd (Wuhan, Hubei, China). Sodium azide was purchased from Zhengzhou painI Chemical Reagent Factory (Zhengzhou, Henan, China). Gelatin, Tween, mouse serum ET-1, NO, NOS3, TXA2, PGI2, vWF, VCAM-1, and TM kits were purchased from Wuhan Jiyinmei Biotechnology Co., Ltd (Wuhan, Hubei, China).

\section{Laboratory equipment}

A micro-high speed centrifuge (Xiangyi H1650-W, 
Changsha, Hunan, China), full wavelength marker (Thermo Multiskan GO, Thermo Fisher Scientific, Waltham, MA, USA), and an automatic animal blood analyzer (Perlong XFA6130, Shanghai, China) were all utilized in this study.

\section{Preparation of anti-mouse platelet serum (APS)}

Whole blood from 160 anesthetized BALB/c mice was collected and PLTs were isolated by gradient centrifugation. The PLTs were then washed twice with phosphate buffered saline (PBS), resuspended, and counted, and the concentration was adjusted to $2.5 \times 10^{6} / \mathrm{mL}$. The anticoagulant was mixed with the same amount of complete and incomplete Freund's adjuvants for antigen preparation. At 0 weeks, the antigen containing complete Freund's adjuvant was injected into the feet, back, and subcutaneous region of guinea pigs, with a total of 5 points $(1 \mathrm{~mL}$ total). The antigen containing incomplete Freund's adjuvant was injected at 1, 2, and 4 weeks according to the same site, dose, and number of injections. At week 5, nonanticoagulant whole blood was taken from the heart of guinea pigs. After standing, guinea pig anti-platelet serum (GP-APS) was obtained by centrifugation and inactivated in a water bath at $56^{\circ} \mathrm{C}$ for $30 \mathrm{~min}$. The red blood cells were adsorbed, and the remaining serum was diluted to a concentration of 1:4 in normal saline for later use.

\section{APS titer detection}

The isolated mouse PLTs were washed 3 times with PBS, and the concentration was adjusted to $6 \times 10^{4} / \mu \mathrm{L}$. Then, $50 \mu \mathrm{L}$ of PLT suspension was added into the microplate hole and centrifuged at $560 \times \mathrm{g}$ for 13 minutes. Buffer solution $(0.2 \%$ gelatin, $0.1 \%$ sodium azide, $0.5 \%$ Tween, $0.15 \mathrm{~mol} / \mathrm{L} \mathrm{PBS}$ ) was added and incubated overnight at $4{ }^{\circ} \mathrm{C}$, followed by a PBS wash. Next, $50 \mu \mathrm{L}$ of serum was added to the PLT suspension and incubated at $37^{\circ} \mathrm{C}$ for 1 hour, followed by a PBS wash. Then, $100 \mu \mathrm{L}$ of enzymelinked A protein was added and incubated at $22{ }^{\circ} \mathrm{C}$ for 45 minutes, followed by 4 washes with PBS. Next, $200 \mu \mathrm{L}$ of p-Nitrophenyl phosphate disodium (dissolved in diethanolamine buffer at a concentration of $1.5 \mathrm{mg} / \mathrm{mL}$ ) was added and incubated at $37{ }^{\circ} \mathrm{C}$ for 30 minutes. Lastly, $50 \mu \mathrm{L}$ of $1 \mathrm{~N}$ sodium hydroxide was added to stop the reaction, and optical density (OD) values were measured by microplate at $405 \mathrm{~nm}$ wavelength.

\section{Modeling and treatment}

Blood was collected from the tail veins of 120 mice, and the PLT count was measured using an automatic blood analyzer. The mice were randomly divided into 6 groups, with 20 in each group, as shown in Figure 1. The APS was injected intraperitoneally at $100 \mu \mathrm{L} / 20 \mathrm{~g}$ once a day until the experiment concluded. Normal saline was used in place of APS in the normal control group. On day 8 after APS injection, all groups except the model group were administered the drugs by gavage at a volume of $0.1 \mathrm{~mL} / 10$ g. After the experiment, all indexes were tested.

Successful animal modeling refers to the following criteria (7): (I) decreased peripheral blood platelet count, (II) injection or other bleeding or spontaneous skin ecchymosis, (III) prolonged clotting time, (IV) bone marrow thromocytogenic megakaryocyte reduction. Meeting three of the above four standards (the first is a necessary standard) was considered to be a successful model.

\section{Peripheral blood PLT counts detection}

Blood was collected from the tail veins of the model mice before and 48 hours after the injection of APS, as well as on day 8 (after intervention of each group began), day 12, and day 15 , respectively, and the peripheral blood PLT counts of the model mice were measured using an automatic blood analyzer.

\section{Classification of the degree of bleeding}

According to the "Clinical Pathway of Immune Thrombocytopenic Purpura" released by the Ministry of Health in 2010 (9), combined with the characteristics of animal experiments, the degree of bleeding was divided into 4 grades as follows: Level 0: no bleeding; Level 1: mild bleeding at the injection site, with other body parts scattered bleeding points; Level 2: obvious bleeding at the injection site, bruises and petechiae in other body parts; Level 3: severe bleeding at the injection site, large amounts of petechiae on the skin, or dark or ulcerated skin. When observing the degree of bleeding, it is necessary to describe the bleeding area and the density of bleeding points, and pay attention to the bleeding site, time of bleeding onset, time of hemostasis, color and shape of the bleeding, and so on. The degree of bleeding was observed on day 8 of GP-APS injection (before medication), day 14 of GP-APS 
Table 1 Changes in peripheral platelet counts of mice in each group $(\bar{x}+\mathrm{s})$

\begin{tabular}{|c|c|c|c|c|c|c|}
\hline Groups & Number of mice & Before injection & 48 hours & Day 8 & Day 12 & Day 15 \\
\hline Model & 20 & $345.50 \pm 36.46$ & $279.33 \pm 70.69^{*}$ & $179.50 \pm 21.06^{*}$ & $183.33 \pm 10.44^{*}$ & $236.00 \pm 9.80^{*}$ \\
\hline Prednisone & 20 & $297.00 \pm 80.95$ & $214.33 \pm 21.70^{\star}$ & $169.83 \pm 38.00^{*}$ & $173.33 \pm 6.77^{\star}$ & $360.83 \pm 93.54^{\Delta \triangle}$ \\
\hline Guipi Decoction & 20 & $467.00 \pm 63.69$ & $146.00 \pm 27.03^{*}$ & $141.00 \pm 20.46^{*}$ & $131.17 \pm 59.95^{\star}$ & $317.83 \pm 61.82^{\triangle}$ \\
\hline Jianpi Shexue & 20 & $335.17 \pm 39.15$ & $156.50 \pm 95.23^{*}$ & $160.17 \pm 59.52^{*}$ & $187.00 \pm 22.08^{*}$ & $342.00 \pm 28.09^{\mathbf{\Delta}} \Delta$ \\
\hline
\end{tabular}

${ }^{*} \mathrm{P}<0.01$ compared with the normal group; ${ }^{\wedge} \mathrm{P}<0.05,{ }^{\triangleright} \mathrm{P}<0.01$ compared with the model group.

injection (the 6th day of medication), and day 16 of GPAPS injection (the end of the experiment).

\section{Vascular active factor detection}

At the end of the experiment, 12 mice in each group were randomly sacrificed via cervical dislocation, and blood was collected from the abdominal aorta. After centrifugation, serum was collected, and the indicators were tested according to enzyme-linked immunosorbent assay (ELISA) kit instructions. Among them, serum from 6 mice was used for the detection of vascular procoagulant active factors (ET-1, TXA2, vWF, VCAM-1), as well as vascular anticoagulant activity factors (NO, NOS3, PGI2, TM). The absorbance (OD value) was measured with an enzyme marker at the wavelength of $450 \mathrm{~nm}$, and the corresponding sample concentration was calculated through the standard curve.

\section{Statistical analysis}

Data were analyzed using SPSS software version 23.0 (IBM Corp., Armonk, Ny, USA), and are presented as mean \pm standard deviation (SD). Enumeration data were analyzed by the chi-squared $\left(\chi^{2}\right)$ test, while measurement data were analyzed by the $t$-test. Differences with a $\mathrm{P}$ value of $<0.05$ were considered statistically significant.

\section{Results}

\section{Antiserum titer detection}

The OD value was measured by microplate at $405 \mathrm{~nm}$ wavelength. The average OD value of the APS serum group was 0.300 , and the average OD value of blank wells was 0.163 , with a difference of about 2 -folds $(\mathrm{P}<0.01)$, indicating that the serum titer was normal.

\section{Peripheral blood PLT counts}

Blood was drawn from the tail veins of 120 mice, diluted, and peripheral blood PLT count was detected by an automatic blood analyzer. Changes in peripheral PLT counts in each group were recorded from the day of APS injection to the 15th day, as shown in Table 1.

Table 1 shows that before the injection of APS, the comparison of PLT counts in the peripheral blood of mice in each group was not statistically significant when analyzed by $t$-test. In addition, from 48 hours to 8 days after the injection of APS, the PLT count in the peripheral blood of mice from each group was statistically significant compared with that of the normal group $(\mathrm{P}<0.01)$. This indicated that the ITP model had been successfully established. Also, the PLT count in the peripheral blood of mice from each group was statistically significant compared with that of the normal group on the 8th day of APS injection (the first administration day) to the 12 th day $(\mathrm{P}<0.01)$, which indicated that the PLT in the peripheral blood of experimental mice was still at a low level. On the 15th day after APS injection (the 8th day of the intervention), the peripheral PLT in mice from the model group was still at a low level compared with that of the normal group $(\mathrm{P}<0.01)$. Further, compared to the normal group, the peripheral PLT of each treated group was not statistically significant when analyzed by $t$-test $(\mathrm{P}>0.05)$, indicating that the peripheral PLT of each treated group could be significantly increased. Among them, the prednisone group and the Jianpi Shexue group were significantly different compared with the Guipi Decoction group and the Jianpi Yiqi group $(\mathrm{P}<0.05)$, indicating that the prednisone group and the Jianpi Shexue group had significant advantages in promoting peripheral blood PLT in model mice.

No serious adverse events were observed in the experimental animals of each treatment group. 
Table 2 The degree of bleeding grade at different time points for each group (number of mice)

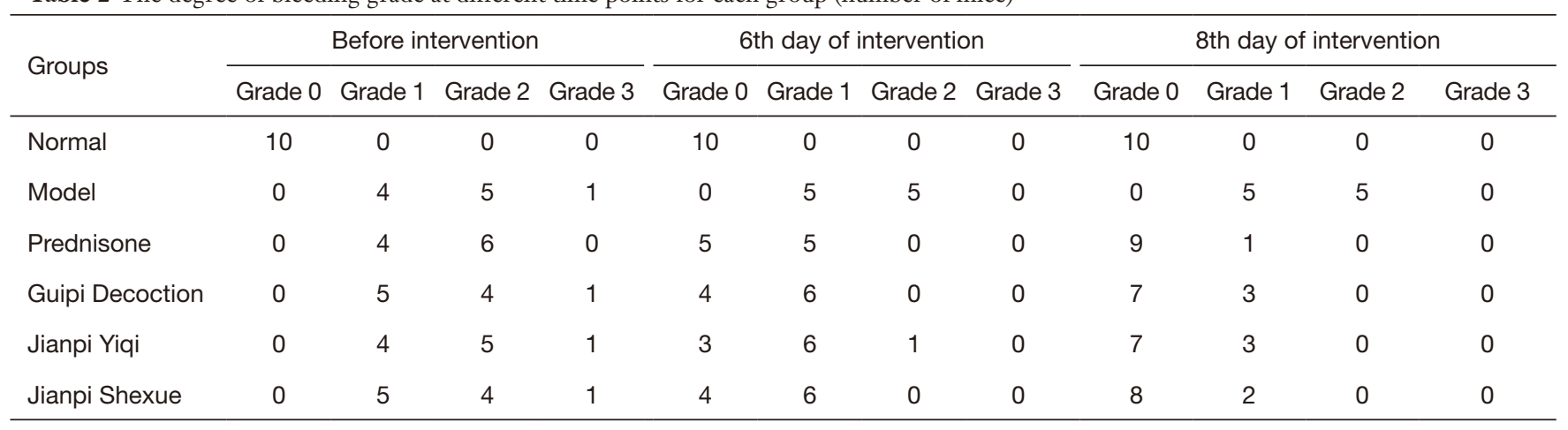

\section{Classification of bleeding degree}

According to the observed time points, the grade of bleeding was taken as the standard, and the bleeding grade changes of 10 mice in each group were observed randomly, as shown in Table 2.

Table 2 shows that on the 8th day of APS injection, the degree of bleeding in each experimental group was comparable with that of the other groups (not including the normal control group) when analyzed by $\chi^{2}$ test $(\mathrm{P}=0.998)$, indicating that the mice in all experimental groups had bleeding tendencies after GP-APS injection. Also, the intervention began on the 8th day of APS injection, and the level of bleeding decreased significantly on day 6 of the intervention, which was statistically significant compared with the model group when analyzed by $\chi^{2}$ test. The largest decrease was seen in the prednisone group, followed by the Jianpi Shexue group, Guipi Decoction group, and Jianpi Yiqi group. There was no statistically significant difference between any 2 groups by $\chi^{2}$ test $(P>0.05)$. Further, on the 8th day of the intervention, the level of bleeding in each group decreased significantly compared with the model group, which was statistically significant by $\chi^{2}$ test $(\mathrm{P}=0.000)$. The greatest decrease in the bleeding grade was seen in the prednisone group $(\mathrm{P}=0.000)$, followed by the Jianpi Shexue group $(\mathrm{P}=0.000)$, Guipi Decoction group $(\mathrm{P}=0.001)$, and Jianpi Yiqi group $(\mathrm{P}=0.001)$. There was no statistically significant difference between any 2 groups by $\chi^{2}$ test $(\mathrm{P}>0.05)$.

\section{Vascular procoagulant active factor analysis}

Vascular procoagulant active factors include vasoconstrictor and procoagulant factors. The results are shown in Table 3.

Table 3 shows that the comparison of ET-1 between the experimental groups and the normal group showed a statistical significance using the $t$-test $(\mathrm{P}<0.01)$, indicating a significant decrease in the detection value. When comparing TXA2, there was a significant increase in each treated group compared with the normal group and the model group, which was statistically significant by $t$-test $(\mathrm{P}<0.01)$. There was no statistically significant difference between any 2 treated groups by $\chi^{2}$ test $(\mathrm{P}>0.05)$. When examining $\mathrm{vWF}$, the difference between the experimental groups and the normal group was statistically significant with $t$-test $(\mathrm{P}<0.01)$, indicating a significant decrease in the detection value. For VCAM-1, the difference between the experimental groups and the normal group was statistically significant by $t$-test $(\mathrm{P}<0.01)$. There were statistically significant differences between "treat the spleen" groups and the prednisone group by $t$-test $(\mathrm{P}<0.01)$, yet there was no statistical significance between the groups $(\mathrm{P}>0.05)$.

\section{Vascular anticoagulant factor}

Vascular anticoagulation factors include vasodilation and anticoagulation factors. The test results are shown in Table 4 .

Table 4 shows that the difference in NO between the experimental groups and the normal group was statistically significant by $t$-test $(\mathrm{P}<0.01)$, indicating a decrease in the detection value. There was no statistically significant difference between any 2 treated groups by $t$-test $(\mathrm{P}>0.05)$. In addition, the test values of NOS3 for each experimental group showed statistical significance compared with those of the normal group $(\mathrm{P}<0.05)$, except for the Guipi Decoction group. There was no statistically significant difference between any 2 of the treated groups by $t$-test $(\mathrm{P}>0.05)$. Compared with the normal group, the PGI2 results of the "treat the spleen" groups were statistically significant when analyzed by $t$-test $(\mathrm{P}<0.01)$. Among the groups, comparisons between the Jianpi Shexue group and 
Table 3 Results of vascular procoagulant factor analysis in each group

\begin{tabular}{|c|c|c|c|c|c|}
\hline Groups & $\mathrm{n}$ & ET-1 (ng/mL) & TXA2 (ng/L) & $v W F(n g / L)$ & $\operatorname{VCAM}-1(\mu \mathrm{g} / \mathrm{L})$ \\
\hline Model & 6 & $52.58 \pm 9.50^{*}$ & $63.16 \pm 11.05$ & $3244.67 \pm 192.55^{\star}$ & $465.37 \pm 121.97^{\star}$ \\
\hline Prednisone & 6 & $40.75 \pm 2.81^{*}$ & $80.30 \pm 8.68^{\star \Delta}$ & $3355.00 \pm 720.50^{*}$ & $317.83 \pm 51.32^{*}$ \\
\hline Jianpi Yiqi & 6 & $43.21 \pm 5.01^{*}$ & $89.99 \pm 20.64^{\star \Delta}$ & $3283.67 \pm 456.29^{*}$ & $658.67 \pm 135.36^{\star \Delta \Delta}$ \\
\hline Guipi Decoction & 6 & $57.18 \pm 10.17^{*}$ & $82.20 \pm 16.31^{\star \Delta}$ & $3058.67 \pm 383.93^{*}$ & $674.97 \pm 181.49^{\star \Delta \Delta}$ \\
\hline
\end{tabular}

${ }^{*} \mathrm{P}<0.01$ compared with the normal group; ${ }^{\Delta} \mathrm{P}<0.01$ compared with the model group; ${ }^{\Delta} \mathrm{P}<0.01$ compared with the prednisone group. ET1, endothelin-1; TXA2, thromboxane A2; vWF, von Willebrand Factor; VCAM-1, vascular endothelial cells, and vascular cell adhesion molecule-1.

Table 4 Results of vascular anticoagulant factor analysis in each group $(\bar{x}+\mathrm{s})$

\begin{tabular}{|c|c|c|c|c|c|}
\hline Groups & $\mathrm{n}$ & $\mathrm{NO}(\mu \mathrm{mol} / \mathrm{L})$ & NOS3 (ng/L) & PGI2 (ng/L) & TM ( $\mu \mathrm{g} / \mathrm{L})$ \\
\hline Model & 6 & $36.87 \pm 11.11^{*}$ & $22.60 \pm 4.65^{\star}$ & $66.24 \pm 11.28$ & $17.44 \pm 3.73^{\star}$ \\
\hline Prednisone & 6 & $32.14 \pm 9.34^{*}$ & $21.30 \pm 5.76^{\star}$ & $60.36 \pm 8.68$ & $19.28 \pm 3.44^{*}$ \\
\hline Jianpi Yiqi & 6 & $38.34 \pm 14.25^{\star}$ & $21.75 \pm 3.68^{\star}$ & $59.19 \pm 11.78^{\Delta}$ & $14.53 \pm 2.32^{\Delta} \Delta$ \\
\hline Guipi Decoction & 6 & $30.59 \pm 9.82^{*}$ & $31.93 \pm 4.60$ & $55.96 \pm 4.33^{\wedge}$ & $17.17 \pm 3.19^{\star}$ \\
\hline
\end{tabular}

${ }^{*} \mathrm{P}<0.05,{ }^{\Delta} \mathrm{P}<0.01$ compared with the normal group; ${ }^{\triangle} \mathrm{P}<0.05$ compared with the model group. NO, nitric oxide; NOS3, nitric oxide synthase 3; PGI2, prostacyclin 2; TM, thrombomodulin.

the model group, prednisone group, Jianpi Yiqi group, and the Guipi Decoction group were statistically significant by $t$-test $(\mathrm{P}<0.05)$. For $\mathrm{TM}$, compared with the normal group, the results of the experimental groups were statistically significant by $t$-test $(\mathrm{P}<0.05)$. There were statistically significant differences between the Jianpi Shexue group and Jianpi Yiqi group and the model group, prednisone group, and Guipi Decoction group by $t$-test $(\mathrm{P}<0.05)$.

\section{Discussion}

ITP can be divided into two types: acute ITP (AITP) and chronic ITP (CITP). Based on the theory of traditional Chinese medicine and combined with clinical manifestations, AITP is more common in children and is a bloodheat syndrome. CITP is more common in adults. It is characterized by slow onset, long course, thrombocytopenia and chronic bleeding. It belongs to the syndrome of splenic failure in controlling blood. It is the most distinctive and advantageous syndrome type in TCM treatment. The
ITP animal model was established by the passive immune modeling method of injecting APS. There are changes in objective detection indicators such as decreased peripheral platelet count, spontaneous hemorrhage at the injection site and skin, prolonged blood clotting time, decreased bone marrow megakaryocytes, weight loss, and shortened swimming time. Meanwhile, they had external characteristics such as reduced activity, slowness of movement, reluctance to eat, reduced diet (drinking), loose stools, withered or dull coat color, and has similar characteristics to human ITP of splenic failure in controlling blood. Therefore, the method of "treated the spleen" was adopted.

Platelets produced by differentiation of bone marrow megakaryocytes have various functions, such as coagulation, hemostasis and repair of damaged vascular endothelial cells. The sugar coating on the surface of PLTs can adsorb plasma proteins and coagulation factor III, and the particles in the PLTs contain coagulation-related substances. When the blood vessel is damaged or ruptured, it will stimulate the platelets to change from a stationary phase to a functional 


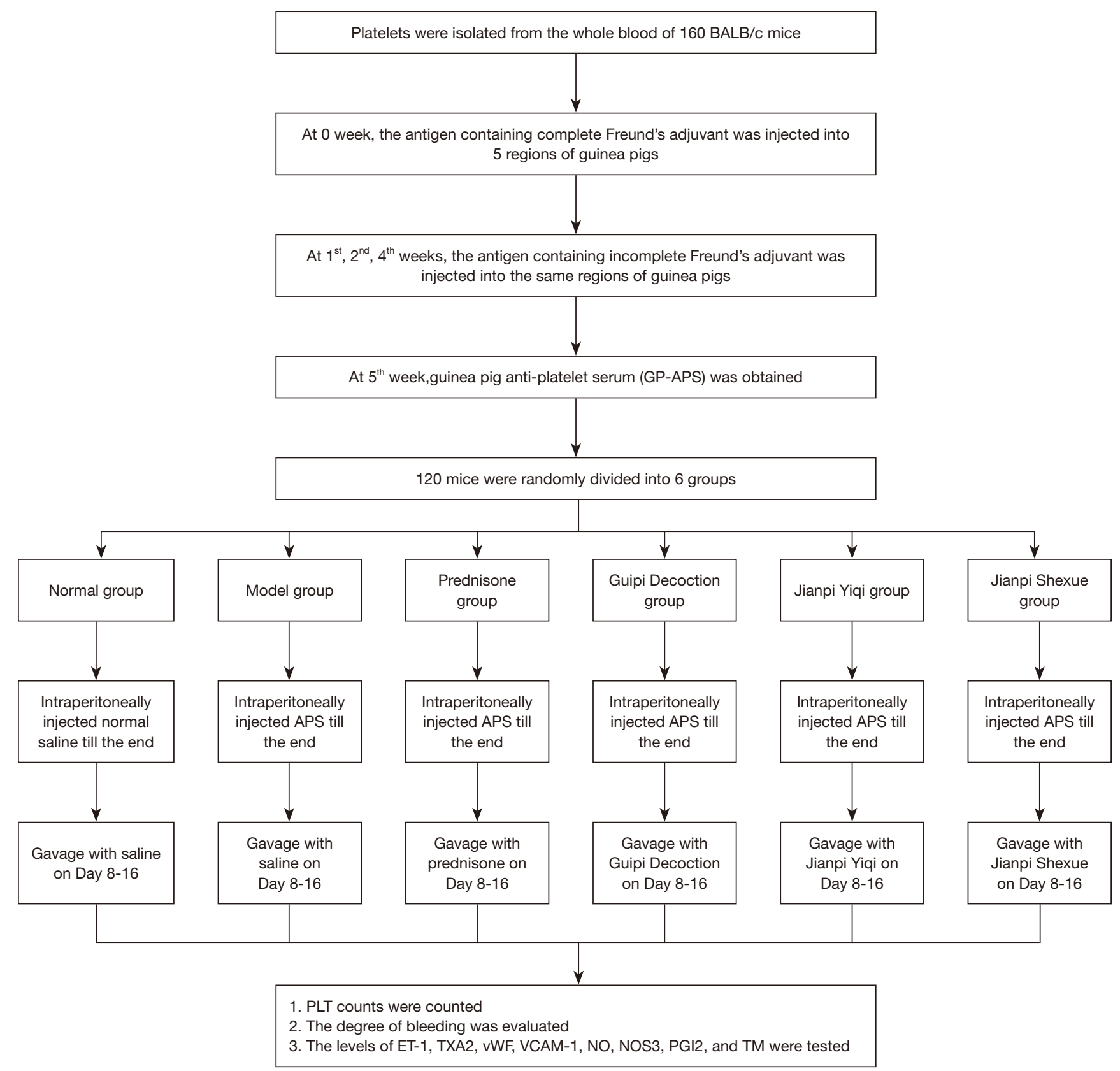

Figure 1 Flow chart of this study.

phase, deformation occurs rapidly, surface viscosity increases and agglomerates. At the same time, under the action of coagulation factor III on the surface of PLTs, the plasma prothrombin becomes thrombin and catalyzes fibrinogen into filamentous fibrin, which together with blood cells form a clot to stop bleeding. In the meantime, PLT particulate matter is released, which further promotes coagulation and hemostasis. Platelets can protect vascular endothelial cells and participate in endothelial cell repair. Therefore, when a patient's peripheral PLT count is less than $50 \times 10^{9} / \mathrm{L}$, there is a risk of bleeding (10), indicating that ITP bleeding is closely related to the amount and quality of PLT in a patient's peripheral blood. In clinical practice, many patients with ITP, especially chronic ITP, have no bleeding tendency, although the peripheral blood PLT count is below $50 \times 10^{9} / \mathrm{L}$, or even below a safe level $\left(30 \times 10^{9} / \mathrm{L}\right)$. 
Obviously, the decrease of peripheral blood PLT in ITP patients is not the only factor for bleeding; it is likely the result of the joint action of multiple links in the coagulation mechanism. Therefore, vascular endothelial cells and their secreted active factors play a vital role (11). Blood vessels are the conduits through which organisms carry blood. In addition to maintaining the exchange of blood and tissues, a large number of active substances exist in normal blood vessels to control the physiological state of blood vessels. In particular, vascular endothelial cells are important sites for the secretion, synthesis, and release of vasoactive substances. These active substances have multiple effects, including relaxing and contracting blood vessels, regulating blood vessel tension, and promoting blood clotting.

Diastolic vasoactive factors are closely related to anticoagulation mechanisms, mainly including prostaglandin (PG), NO and NOS3, and TM. Blood coagulation is affected by PG, and its metabolized derivatives such as prostacyclin (PGI2), mainly via vasodilation, inhibiting platelet aggregation, and regulating platelet activation (12). Both NO and NOS3 mainly act by relaxing smooth muscle cells and expanding blood vessels, quickly penetrating the cell membrane to diffuse into the blood in order to act on PLT cells and reduce PLT activity, inhibiting agglutination and adhesion to vascular endothelium (13). As an important intravascular coagulation inhibitor, TM changes thrombin from a procoagulant factor to an anticoagulant factor, thus, mainly regulating the body's coagulation and anticoagulation dynamic balance (14).

Systolic vasoactive factors are closely related to the coagulation mechanism and mainly include endothelin (ET), thromboxane A2, Von Willebrand factor, and vascular endothelial cell adhesion molecule-1. An important factor that regulates cardiovascular function, ET plays an important role in maintaining vascular tone and the homeostasis of the cardiovascular system. Among them, ET-1 is by far the strongest vasoconstrictive factor. It can cause the contraction of blood vessels, especially small blood vessels, which is beneficial to blood coagulation and hemostasis (15). Another strong vasoconstrictor, TXA2 activates and aggregates platelets, which is helpful for repairing tissue damage and hemostasis (16). The multimeric glycoprotein $\mathrm{vWF}$ is an adhesive that binds to the PLT membrane GPIb-IX complex and subendothelial collagen, mediates platelet adhesion at vascular injury sites, and binds to factor VIII to effectively stop bleeding (17). In addition, vWF can also bind to GPIIbIIIa and participate in the PLT aggregation process. The protein VCAM-1 is a cell adhesion molecule that is mainly involved in the interaction between cells, and between cells and the extracellular matrix. Reduced activity can lead to increased vascular permeability, tissue ischemia and hypoxia, and increased blood extravasation (18).

The vasomotor activity that controls vascular tone is a contradictory coordination of factors. It is generally believed that vasodilating active factors have an anticoagulant effect, which is not only a key factor in preventing thrombosis, but also an important substance that affects blood coagulation and hemostasis. Conversely, vasoconstriction active factor has the effect of promoting coagulation, which is a risk factor for thrombosis, as well as the key to promoting blood clotting and hemostasis. The dynamic balance of these 2 factors plays various roles in different diseases. When the body develops thrombocytopenia, especially ITP, the disordered immune mechanism can lead to vascular endothelial cell damage, an imbalance of vascular secretion, synthesis and release of vasoactive factors, and can directly or indirectly affect vasomotor activity and coagulation. Therefore, effective regulation of vasomotor activity can prevent the risk of bleeding or achieve hemostasis in patients with low PLT values. Based on this, we established precepts of "treating from the spleen" for ITP in clinical practice (19) and formulated the Jianpi Shexue recipe. Early clinical trials have shown that this prescription can significantly increase the peripheral blood PLT counts of ITP patients and can improve bleeding and related clinical symptoms (20-21). Animal experiments have also confirmed that the prescription can improve peripheral blood PLT counts, has good hemostatic effects in model mice (22-25), and clarified some of the effective mechanisms (26-28). In order to further explore the mechanism of ITP on "treating the spleen", we used the Jianpi Shexue recipe (Sijunzi Decoction plus Astragalus, Ejiao, and Madder root (Qian Cao Gen)) as the standard observation drug, and selected the Jianpi Yiqi recipe (Sijunzi Decoction plus Astragalus Decoction) and Guipi Decoction together as the "treat the spleen" formula; prednisone was chosen as a control drug. We then observed the effects of the 3 "treat the spleen" formulae on the peripheral blood PLT count, hemorrhage grading, and vasoactive factors in ITP animal models. When comparing values from 48 hours to 8 days after the injection of APS (dose timepoint), the peripheral blood PLT counts of the mice in the experimental groups significantly decreased compared with the normal group, and the difference was statistically significant by $t$-test $(\mathrm{P}<0.01)$. On the 8 th day of administration, compared with the model group, the peripheral blood PLT counts 
of the mice in each group were significantly increased by $t$-test $(\mathrm{P}<0.05)$. Among them, the differences between the prednisone group and Jianpi Shexue group compared with the Guipi Decoction group and Jianpi Yiqi group were significant by $t$-test $(\mathrm{P}<0.05)$, indicating that the prednisone group and the Jianpi Shexue group have obvious advantages in improving peripheral blood PLT in model mice. Additionally, the results showed a hemostatic effect. On the 8th day of injection of APS (dose time point), except for the normal group, the experimental groups had different degrees of bleeding tendencies. On the 6th and 8th days of drug intervention, compared with the model group, the bleeding grade of each group was significantly decreased. The descending magnitudes were in the order of the prednisone, Jianpi Shexue, Guipi Decoction, and Jianpi Yiqi group, respectively. The comparison among the groups was not statistically significant. When looking at results for the vascular procoagulant factor, the ET-1 detection value of each group in the experiment was significantly lower than that of the normal group. The TXA2 test values for each treated group were significantly increased compared with the normal group and the model group. The vWF and VCAM-1 test values of each experimental group were significantly lower than those of the normal group. Furthermore, the VCAM-1 of the "treat the spleen" groups were increased compared with the prednisone group and the model group, showing that "treat the spleen" therapy has the advantage of improving VCAM-1. The vascular anticoagulant factor results showed that when compared with the normal group, the NO detection value in each group of the experiment decreased significantly, while the comparison between groups was not statistically significant. Except for the Guipi Decoction group, the NOS3 test value in each experimental group was significantly lower than that in the normal group, and the comparison between groups was statistically significant. The PGI2 test values of the "treat the spleen" groups were significantly lower than those of the normal group. Among them, the decrease was obvious in the Jianpi Shexue group compared with the model, prednisone, Jianpi Yiqi, and Guipi Decoction groups, respectively. Compared with the normal group, the TM detection values in each experimental group were significantly lower. Among them, the values of Jianpi Shexue group and Jianpi Yiqi group were significantly lower than those of the model, prednisone, and the Guipi Decoction groups. These results lead to the conclusion that in addition to increasing the peripheral blood PLT count and reducing hemorrhage grade in ITP model mice, the "treat the spleen" recipes can increase the vascular procoagulant factors TXA2 and VCAM-1, and downregulate PGI2 and TM detection values. Adjusting the dynamic balance of procoagulant and anticoagulant active factors may be one of the effective mechanisms of hemostasis.

\section{Acknowledgments}

Funding: This work was supported by National Program on Key Basic Research Project (973 Program) (2013CB531705), Youth Program of National Natural Science Foundation of China (81703903; 81803904), Youth Program of Zhejing TCM Science and Technology Program (2018ZQ019), and University Research Fund Project of Zhejing Chinese Medical University (2018ZG02).

\section{Footnote}

Reporting Checklist: The authors have completed the ARRIVE reporting checklist. Available at http://dx.doi. org/10.21037/apm-21-765

Data Sharing Statement: Available at http://dx.doi. org/10.21037/apm-21-765

Conflicts of Interest: All authors have completed the ICMJE uniform disclosure form (available at http://dx.doi. org/10.21037/apm-21-765). The authors have no conflicts of interest to declare.

Ethical Statement: The authors are accountable for all aspects of the work in ensuring that questions related to the accuracy or integrity of any part of the work are appropriately investigated and resolved. Experiments were performed under a project license (No.: XYLS2019069) granted by ethics board of Medical Ethics Committee of Xi' an Medical College, in compliance with Chinese guidelines for the care and use of animals.

Open Access Statement: This is an Open Access article distributed in accordance with the Creative Commons Attribution-NonCommercial-NoDerivs 4.0 International License (CC BY-NC-ND 4.0), which permits the noncommercial replication and distribution of the article with the strict proviso that no changes or edits are made and the original work is properly cited (including links to both the formal publication through the relevant DOI and the license). See: https://creativecommons.org/licenses/by-nc-nd/4.0/. 


\section{References}

1. Xiong TT, Xie XT. The latest research progress on the cell immunity pathogenic mechanism of Immune Thrombocytopenia. J China Pediatr Blood Cancer 2019;24:321-7.

2. Kühne T, Berchtold W, Michaels LA, et al. Newly diagnosed immune thrombocytopenia in children and adults: a comparative prospective observational registry of the intercontinental cooperative immune thrombocytopenia study group. Haematologica 2011;96:1831-7.

3. Liu YM, Zhou YF. Research progress of traditional Chinese medicine in treating primary immune thrombocytopenia. Chinese Journal of Ethnomedicine and Ethnopharmacy 2018;27:57-9.

4. Wu YX, Yuan Z, Ma XH, et al. Clinic Observation on Idiopathic Thrombocytopenic Purpura by Treatment from Spleen. World Chinese Medicine 2016;11:1782-5.

5. Li TT, Hou L, Zhang YY, et al. Theoretical basis and clinical practice of treating immune thrombocytopenia from the spleen. Beijing Journal of Traditional Chinese Medicine 2015;34:304-6.

6. Wang HL, Liu BS. Research Progress on the Establishment of Animal Model with Immune Thrombocytopenic Purpura. Journal of Experimental Hematology 2018;26:273-7.

7. Lang HY, Chu YT, Ma W, et al. Disease - syndrome Combined Animal Models for Immune Thrombocytopenic Purpura. Information on Traditional Chinese Medicine 2017;34:39-43.

8. He H, Xu Y, Qin B, et al. Establishment and Evaluation of Animal Models of Immune Thrombocytopenic Purpura. Progress in Modern Biomedicine 2016;16:1971-4.

9. Chen R. Clinical pathway of immune thrombocytopenic purpura (2010 Edition). Chinese Community Doctors 2011;27:17.

10. Yu GC. Analysis of 22 cases of ITP clinical bleeding risk. Jiangxi Medical Journal 2010;45:801-2.

11. Li W, Zhang MJ, Wang Y, et al. The relationship of platelet activating factors and vascular endothelial activity markers to lacunar infarction. West China Medical Journal 2019;34:1143-7.

12. Cai DZ, Zhang X. Efficacy of Danggui on TCM syndromes, serum inflammatory factors, plasma thromboxane and prostacyclin levels in patients with essential hypertension. Clinical Journal of Chinese Medicine 2019;11:37-9.

13. Quan SD, Yu YJ, Liang ZX. Effect of NO inhalation combined with milrinone on hemodynamics and platelet aggregation in neonates with persistent pulmonary hypertension. Journal of Clinical and Experimental Medicine 2020;19:89-91.

14. Xu J, Ding YN, Yang M, et al. Joint Detection of D-dimer And Activated Partial Thromboplastin time, Thrombomodulin for Breast Cancer Patients after Surgery Deep Vein Thrombosis Value. Chinese Journal of Thrombosis and Hemostasis 2019;25:952-3.

15. Wu QX, Wang P, Wang DZ, et al. Dynamic Study on Changes of Vascular Endothelial Active Substances in Mice Model of Immune Thrombocytopenia with Syndrome of Qi Failing to Control Blood. Chinese Journal of Information on TCM 2019;26:56-61.

16. Zhang Y, Liu HX, Kang QF, et al. Effects of optimum Shenyuandan Decoction on insulin resistance, serum TXA2/PGI2 ratio and DNA methylation level of mice with atherosclerosis. Chinese Journal of Information on TCM 2018;25:53-7.

17. Chang FY, Feng ZR, Wang ZM. Changes of serum vWF, GDF-15, PCSK-9, and APN in patients with coronary heart disease and its relationship with the degree of coronary artery disease. Chinese Journal of Integrated Medicine on Cardiocerebrovascular Disease 2019;17:3570-3.

18. Zhu XH, Ding QC, Lai SL, et al. Effect of autophagy on inflammatory factors - induced vascular cell adhesion molecule 1 expression. Modern Preventive Medicine 2019;46:2419-23.

19. Wu XY, Chen GL, Wang YL, et al. Treatment of Immune Thrombocytopenia Based on Spleen Controlling Blood. Liaoning Journal of Traditional Chinese Medicine 2017;44:659-61.

20. Wang J, Zhang Y, Zhang L, et al. Clinical curative effect observation on treatment of immune thrombocytopenia treated with Jianpi Yiqi Shexue Granules. China Journal of Traditional Chinese Medicine and Pharmacy 2018;33:5700-4.

21. Lang HY, Zhang L, Wang J, et al. Clinical Research on Specific Immune Function of Immune Thrombocytopenia Treated by Jianpi Yiqi Shexue Granule. Journal of Medical Research 2019;48:45-9.

22. Li X, Zhu CL, Zhao N, et al. Preventive and therapeutic effects of Jianpi Yiqi Shexue Keli and its disassembled prescriptions in treating zebrafish cerebral hemorrhage induced by simvastatin. Journal of Beijing University of Traditional Chinese Medicine 2017;40:1004-10.

23. Zhang YY, Tian SD, Qi S, et al. Study on the blood flow 
effect of Invigorating Spleen, Benefiting Qi, Controlling Blood Formula and its decomposed recipes on Simvastatin induced zebrafish cardiac hemorrhage model. China Medical Herald 2017;14:9-12.

24. Chu YT, Zhu XY, Zhang YY, et al. Hemostatic Effect of Spleen-invigorating, Qi-replenishing and Bloodcontaining Formula on Simvastatin-induced Zebrafish Hemorrhage Model. Journal of Experimental Hematology 2017;25:853-9.

25. He H, Sun YP, Zheng L, et al. Effect of Jianpi Yiqi Shexue Prescription on the Peripheral Hemogram of ITP Mice Models. Acta Chinese Medicine and Pharmacology 2015;43:22-4.

Cite this article as: Zhang Y, Jiang M, Wang J, Gao J, Guo M, Liu J, Chen X, Lang H. The hemostatic mechanism of "Treated the Spleen" therapy on immune thrombocytopenia based on the characteristics of vasoactive factors. Ann Palliat Med 2021;10(4):4612-4622. doi: 10.21037/apm-21-765
26. Chen K, Dai XY, Zhao N, et al. Study on Relationship Between Spleen Failing to Control Blood Related Diseases and Blood Neurotransmitter Changes. Liaoning Journal of Traditional Chinese Medicine 2018;45:245-8.

27. Xu Y, He H, Zheng L, et al. Impacts of Jianpi Shexue Formula on the Expression of Serum Neurotransmitter in ITP Mice, World Journal of Integrated Traditional and Western Medicine 2017;12:928-32.

28. He H, Xu Y, Zheng L, et al. Effect of Jianpi Yiqi Shexue Prescriptionon SIgA and $\beta$-EP in Serum from Mice Models of ITP. Information on Traditional Chinese Medicine 2015;32:47-9. 